\title{
A Formação Continuada de Agentes Comunitários de Saúde Através de Redes Sociais: resultados preliminares
}

\author{
Antonio Augusto Fidalgo Neto ${ }^{1}$, Daniel José Garcia dos Santos de Faria ${ }^{1}$, \\ Luiz Anastacio Alves ${ }^{1}$, Renato Matos Lopes ${ }^{1}$ \\ ${ }^{1}$ Laboratório de Comunicação Celular, Instituto Oswaldo Cruz) - Fundação Oswaldo Cruz. \\ Av.Brasil, 4365 - Manguinhos, Rio de Janeiro - CEP:21040-360 - Brasil. \\ fidalgoaa@gmail.com, daniel.lcc.fiocruz@gmail.com, \\ alveslaadioc.fiocruz.br, renatomlefiocruz.ioc.br
}

\begin{abstract}
The Community Health Worker (CHW) has a vital importance for the Family Health Strategy (FHS). On the other hand, literature shows a gap in CHW education as well as in their professional education. This paper shows an in-running project, which aims to develop resources for the professional education of CHW using the social networks. Among the results, there is a Facebook ${ }^{\circledR}$ webpage and some video material addressing important issues for CHW. Some of these videos are already available on YouTube ${ }^{\circledR}$. This content will be used as complementary educational resources with the $\mathrm{CHW}$ on Facebook ${ }^{\circledR}$ webpage. A convenient sample from $15 \mathrm{CHW}$ of the Cachoeiras de Macacu, a small village located in the state of Rio de Janeiro, were recruited to perform a preliminary assessment of three videos produced by the project. The content presented was the vaccination, the intestinal protozoa (some waterborne diseases) and the pediculosis. The evaluations were performed by psychometric Likert scale. All three videos obtained averages quite satisfactory on the content, professional importance and didactic. Thus, it is possible to infer a great potential of the use of this content through social networks. Besides the production these relevant content on topics of the Public Health, focused on the professional education of the CHW, the development of this project search to evaluate the potential application of social networks for Education in Health.
\end{abstract}

Resumo: O Agente Comunitário de Saúde (ACS) possui importância vital para a Estratégia de Saúde da Família (ESF). Entretanto, a literatura aponta fragilidades na sua formação, assim como para a existência de uma demanda à formação continuada desses profissionais. O presente trabalho apresenta um projeto em desenvolvimento que se propõe a elaborar recursos e processos de ensino e aprendizagem para a formação continuada dos ACS através das redes sociais. Dentre os resultados obtidos, existe a "página-plataforma" no Facebook ${ }^{\circledR}$, assim como material em vídeo abordando temas importantes e de interesse dos ACS. Alguns vídeos já estão disponibilizados no Youtube ${ }^{\circledR}$. Esses e outros materiais serão utilizados como recursos educacionais complementares no momento em que cada tema for trabalhado com os ACS no Facebook ${ }^{\circledR}$. Nesse sentido, contatos realizados entre a equipe do projeto e a Secretaria de Saúde do município de Cachoeiras de Macacu, situado no Estado do Rio de Janeiro, possibilitou que 15 agentes fizessem uma avaliação preliminar de três vídeos produzidos pelo projeto. Os temas avaliados foram vacinação; protozooses intestinais (algumas doenças veiculadas pela água) e pediculose. As avaliações foram realizadas através da escala psicométrica de Likert. Os três vídeos obtiveram médias bastante satisfatórias sobre o conteúdo, importância e didática. Desta forma é possível inferir sobre um grande potencial do uso deste tipo de conteúdo através das redes sociais. Além da produção de conteúdos de qualidade sobre temas do Campo da Saúde Pública, voltados à formação continuada dos ACS, o desenvolvimento deste projeto tem como perspectiva futura realizar estudos para avaliar o potencial de aplicação das redes sociais para o Ensino em Saúde.

Palavras-Chave: Agentes Comunitários de Saúde, Formação Continuada, Redes Sociais, Facebook, YouTube. 


\section{Introdução}

Os mais de 230 mil Agentes Comunitários de Saúde (ACS) que desenvolvem suas atividades nos mais de 5000 municípios brasileiros, segundo dados do Departamento da Atenção Básica do Ministério da Saúde ${ }^{1}$, possuem um papel fundamental no modelo de Atenção Básica em Saúde no Brasil, atuando como membro das equipes de Estratégia de Saúde da Família (ESF). Contudo, a formação dos ACS é complexa ao exigir um profissional com conhecimentos biológicos e de saúde, incluindo também o perfil de líder social com a capacidade de mobilizar a comunidade (Bornstein, \& Stotz, 2008; Santos et al., 2011; Marzari et al., 2011). A Lei 11.350 do dia 05 de outubro de 2006, que rege as atividades dos ACS, no seu Artigo $3^{\circ}$, considera as seguintes atividades desses profissionais na sua área de atuação:

\section{I - a utilização de instrumentos para diagnóstico demográfico e sociocultural da comunidade; \\ II - a promoção de ações de educação para a saúde individual e coletiva; \\ III - o registro, para fins exclusivos de controle e planejamento das ações de saúde, de nascimentos, óbitos, doenças e outros agravos à saúde; \\ IV - o estímulo à participação da comunidade nas políticas públicas voltadas para a área da saúde; \\ $V$ - a realização de visitas domiciliares periódicas para monitoramento de situações de risco à família; $e$ \\ VI - a participação em ações que fortaleçam os elos entre o setor saúde e outras políticas que promovam a qualidade de vida.}

Nesse sentido, é fundamental a constante reflexão sobre o exercício das funções e da melhoria da qualidade dos serviços prestados por esses profissionais, assim como o desenvolvimento de processos educativos que os auxiliem através de processos de formação continuada, visto que relatos da literatura apontam deficiências para a sua formação. Nunes e colaboradores (2002) demonstraram insatisfação de um grupo de ACS em relação ao processo de formação que receberam. Os autores sugerem que o processo de integração entre os demais profissionais da saúde, médicos e enfermeiros, gera ansiedade pela comparação entre as formações, gerando uma demanda por formação continuada dos ACS. Marzari e colaboradores (2011) também apontam para deficiências na formação dos ACS, assim como para a inexistência de ações sistemáticas de atualização dos conhecimentos desses profissionais.

Optamos no uso de redes sociais pelo fato das mesmas possibilitarem um grande potencial de comunicação e de colaboração profissional (Cheung, Chiu, \& Lee, 2011). As redes sociais vêm sendo utilizadas como importantes ferramentas educacionais (Veletsianos \& Kimmons, 2013), possibilitando ao mesmo tempo a utilização de vários recursos e com grande potencial de alcance geográfico. Nos EUA, por exemplo, dados apontam que mais de $90 \%$ dos estudantes do equivalente ao ensino médio brasileiro usam algum tipo de rede social, e dentre os vários objetivos a troca de informações envolvendo o ensino (Veletsianos, Kimmons, \& French, 2013). Ademais, as redes sociais podem apresentar um grande potencial para fins educacionais, considerando que as mesmas são de grande alcance, democráticas, flexíveis e altamente dinâmicas (Madhusudhan, 2012).

O Facebook ${ }^{\circledR}$ no ano de 2013 alcançou mais de 1 bilhão de usuários ativos ao redor do mundo (Facebook, 2013) e o Brasil ocupa o terceiro lugar em registros ativos nesta rede, com um número próximo a 80 milhões de usuários, enquanto os Estados Unidos da América e a Índia ocupam, respectivamente, as duas primeiras posições no ranking (TheEconomicTimes, 2013). Além disso, a pesquisa científica e a geração do conhecimento através de estudos com

\footnotetext{
${ }^{1}$ http://dab.saude.gov.br/portaldab/
} 


\section{CBIE-LACLO 2015}

Anais do XXVI Simpósio Brasileiro de Informática na Educação (SBIE 2015)

a aplicação de redes sociais vêm aumentando de modo significativo nos últimos anos. Uma busca simples no site Web of Science ${ }^{2}$ usando a palavra Facebook, apenas no campo "titulo", nos últimos cinco anos, apresenta mais de 1820 resultados de produtos. A busca com a palavra Youtube ${ }^{\circledR}$, com as mesmas condições mencionadas, gera mais de 430 produtos.

A partir do panorama apresentado, está em desenvolvimento um projeto de uso de redes sociais, especialmente o Facebook $^{\circledR}$ e o Youtube $^{\circledR}$, na geração de recursos e de desenvolvimento de processos de ensino e aprendizagem direcionados para a formação continuada de ACS. Com um maior detalhamento, são dois objetivos principais que se busca com o projeto, ou seja: 1) produzir e distribuir, através das Redes Sociais, recursos educacionais de qualidade sobre diversos temas e assuntos de Saúde Pública para a formação continuada de Agentes Comunitários de Saúde vinculados com Programas de Estratégia de Saúde da Família e 2) avaliar o potencial do Facebook e do YouTube, como plataformas de ensino e aprendizagem, numa perspectiva de aplicação de metodologias ativas de ensino, tais como a Problematização e a Aprendizagem Baseada em Problemas, tendo como público alvo os ACS. A hipótese é que a partir de um planejamento cuidadoso de ensino, fundamentado no uso de Metodologias Ativas de Ensino, e de demandas e necessidades dos próprios profissionais que atuam no Campo da Saúde pública, o uso de redes sociais pode ser muito útil na formação continuada dos ACS, possibilitando também a aprendizagem significativa de temas relacionados com a Saúde Pública e a Atenção Básica em Saúde.

\section{Procedimentos Metodológicos}

\subsection{Elaboração de Conteúdos e de Temas Para a Formação Continuada}

Os conteúdos ou temas que serão desenvolvidos na formação continuada estão ou serão produzidos e discutidos, primordialmente, a partir de demandas dos próprios ACS e dos gestores da Estratégia da Saúde da Família. Nesse sentido, conversas já estão sendo realizadas entre a equipe do projeto e a Secretaria de Saúde do município de Cachoeiras de Macacu, situado no Estado do Rio de Janeiro, com o intuito de viabilizarmos um convênio e desenvolvermos atividades educacionais com os ACS que desenvolvem suas atividades naquele município. Nos próximos meses, será desenvolvido um estudo com sessenta ACS que desenvolvem suas atividades naquele município. Além desses profissionais, os gestores das ESF do município irão responder um questionário que irá apontar temas ou conteúdos importantes e necessários para serem desenvolvidos para a formação continuada. Todo o conteúdo será realizado através dos diversos meios que a Tecnologia da Informação e Comunicação propicia, como por exemplo, textos hipermídia, objetos de aprendizagem e, principalmente, videoaulas.

\subsection{Avaliação Preliminar das Videoaulas Produzidas}

Foi desenvolvida uma dinâmica de duas horas para a realização de uma avaliação preliminar de três das videoaulas já elaboradas: 1) Vacinação; 2) Protozooses Intestinais e 3) Pediculose. A dinâmica da apresentação ocorreu em uma sala com equipamentos para a transmissão das videoaulas em um telão. Após a divulgação de cada vídeo os agentes receberam um questionário contendo 16 afirmativas que deveriam ser preenchidas em até 20 minutos. A avaliação foi realizada de acordo com a escala psicométrica de Likert contendo 5 opções para resposta. Para cada afirmativa do questionário, os ACS tinham a opção de escolher: 1) discordo muito; 2) discordo; 3) não concordo e nem discordo; 4) concordo e; 5) concordo muito. O resultado dos três vídeos foram compilados em médias e seus respectivos erros sendo utilizado o software estatístico GraphPad Prism v. 5.0 ${ }^{3}$.

\footnotetext{
${ }^{2}$ http://apps.webofknowledge.com/

3 http://www.graphpad.com/scientificsoftware/
} 


\section{Resultados e Discussão}

Dentre os resultados obtidos, existe a "página-plataforma" no Facebook ${ }^{\circledR}$, disponível em https://www.facebook.com/educaacs, assim como material em vídeo abordando temas importantes e de interesse dos ACS. Alguns desses recursos estão disponíveis no Youtube ${ }^{\circledR}$, através do link https:/www.youtube.com/user/canallcc. Dentre os conteúdos iniciais produzidos, estão disponíveis: 1) "O Caminho das Águas"; 2) "Reflexões Sobre a Estratégia de Saúde da Familia e a Importância do ACS"; 3) "Hepatite A"; 4) "Vacinação"; 5) "Diabetes"; 6) "Pediculose"; 7) "Doenças Veiculadas por Água"-Parasitoses Intestinais, 6) "Pediculose" e 7) "Toxoplasmose". Além dessas videoaulas, foram elaborados um vídeo de apresentação do projeto e outro de apresentação da proposta pedagógica a ser desenvolvida no processo de ensino e aprendizagem.

Os vídeos sobre vacinação, protozooses intestinais e pediculose foram avaliados pelos 15 ACS e os resultados são apresentados na Figura 1. Os agentes comunitários concordaram com a importância dos vídeos produzidos para a sua formação, assim como na adequação dos mesmos para a suas realidades de trabalho. Estudos realizados com grupos de ACS apontaram grande demanda por formação continuada (Marzari, 2011). No presente estudo, o conjunto de afirmativas apresentadas para avaliação através da escala de Likert possibilitou a mesma inferência de Marzari (2011). A grande utilidade dos conteúdos apresentados, assim como o aspecto de incentivo ao aprofundamento dos assuntos abordados, corroboram pela busca por formação continuada dos ACS (Figura 1). É importante ratificar que os conteúdos desenvolvidos - e a desenvolver - foram resultado de consulta prévia de um grupo representativo de ACS. Isso reafirma o interesse desse grupo de profissionais pelo seu contínuo desenvolvimento e melhoria de suas habilidades.

No conjunto de afirmativas apresentadas aos ACS, três apresentavam padrões contrários de raciocínio de resposta. Quando foram solicitados a avaliar se o "conteúdo foi apresentado superficialmente" e "sobre a duração dos vídeos" (muito curto ou longo), os ACS deveriam alterar os padrões de resposta de "concordo muito" ou "concordo", para "discordo" ou "discordo muito", caso continuassem com as avalições positivas. A partir dos resultados obtidos foi possível assegurar maior confiança nas avaliações considerando que houve o preenchimento na mesma lógica das anteriores, ou seja, concordo com os aspectos positivos do conteúdo apresentando, os ACS discordaram ou discordaram muito que os vídeos produzidos eram ou muito curtos, ou excessivamente longos (Figura 1).

Como mencionado anteriormente, o perfil profissional esperado para um ACS é complexo, demandando várias competências e habilidades. Os ACS possuem, em geral, formação de nível médio, o que traz também a necessidade de uma abordagem adequada. Ao longo do planejamento e execução deste estudo houve a preocupação no sentido de tornar a comunicação acessível. De acordo com a avaliação dos ACS a linguagem utilizada foi clara para a grande maioria, onde mais de $90 \%$ deles "concordaram" ou "concordaram muito" sobre a afirmativa. Da mesma forma, um número expressivo de agentes "concordaram" ou "concordaram muito" que os conteúdos foram apresentados de forma clara. A comunicação clara pode contribuir ainda mais para o grande potencial das redes sociais na educação.

Ademais, as médias para todos os itens avaliados foram consistentemente positivas em relação aos parâmetros ou afirmativas existentes no questionário aplicado (Figura 1). De forma subjetiva, também foi constatado um grande interesse dos ACS pelos temas das videoaulas, assim como uma grande motivação dos mesmos em participar da atividade proposta. No seu conjunto, esses dados reforçam outras informações da literatura que apontam que esses profissionais possuem interesse para processos educacionais de formação continuada (Nunes, Trad, Almeida, Homem, \& Melo, 2002; Mota \& David, 2010). 
CBIE-LACLO 2015

Anais do XXVI Simpósio Brasileiro de Informática na Educação (SBIE 2015)

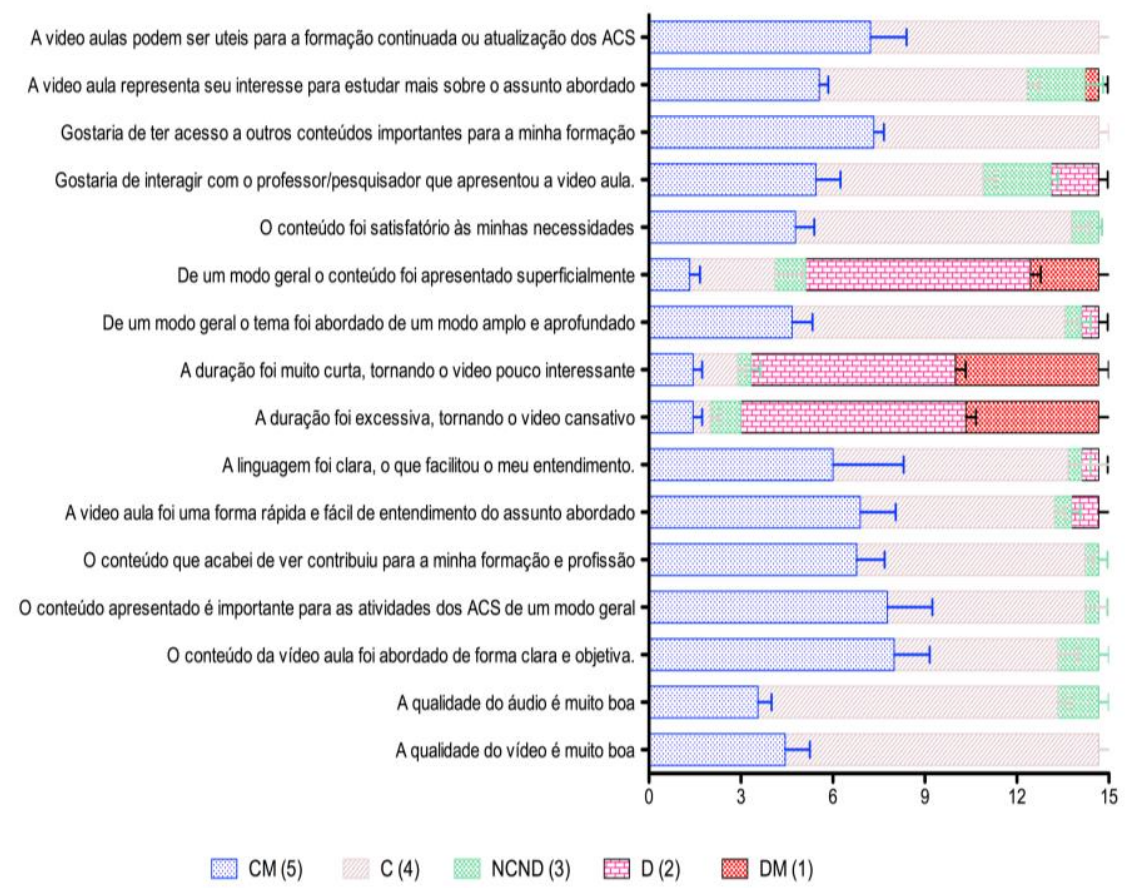

Figura 1. Escala psicométrica de Likert contendo respostas de quinze ACS. Os resultados são apresentados como as médias \pm o erro padrão da avaliação de 3 videoaulas. $\mathrm{CM}$ - concordo muito; $\mathrm{C}$ - concordo; NCND não concordo nem discordo; D - Discordo; DM - discordo muito.

\section{Considerações Finais}

Novos estudos serão realizados para que se possa investigar o potencial de uso das redes sociais como plataformas de ensino em saúde, incluindo também a utilização de estratégias ativas de ensino como a Problematização e a Aprendizagem Baseada em Problema (ABP ou PBL de Problem-Based Learning) que, dentre outros aspectos pedagógicos, valorizam a realidade do trabalho exercido por profissionais de saúde (Mitre et al., 2008). Além da produção de recursos educacionais e de cursos à distância para os ACS, através do Facebook e do YouTube, o prosseguimento das atividades de ensino e pesquisa irão contribuir para a literatura em Ensino em Saúde tendo como enfoque o uso das redes sociais como ferramentas ou ambientes de aprendizagem.

\section{Agradecimentos}

Os autores agradecem a FAPERJ, ao convênio Fiocruz - Capes de apoio ao "Plano Brasil sem Miséria" e a Secretaria de Saúde da prefeitura da cidade de Cachoeiras de Macacu.

\section{Referências}

Bornstein, Vera Joana, \& Stotz, Eduardo, Navarro. (2008). Concepts involved in the training and work processes of community healthcare agents: A bibliographical review. Ciência e Saúde Coletiva, 13(1), 9.

Cheung, C. M. K., Chiu, P. Y., and Lee, M. K. O. (2011). Online social networks: why do students use facebook? Computers in Human Behavior, 27(4), 1337-1343.

Facebook. (2013). Press room statistics. Retrieved 27/06/2013, 2013, from https://newsroom.fb.com/Key-Facts

Madhusudhan, Margam. (2012) Use of social networking sites by research scholars of the university of delhi: a study. The International Information \& Library Review, 44, 13.

Marzari, C. K., Junges, J. R., and Selli, L. (2011) Community Health Agents: profile and education. Cien Saude Colet, 16 Suppl 1, 873-880.

Mitre, S.M., Siqueira-Batista, R., Girardi-deMendonça, J.M., De Morais-Pinto N.M., Meirelles, C.D.B., Pinto-Porto,C.T., Hoffmann, L.M.A. (2008). Metodologias ativas de ensino-aprendizagem na formação profissional em saúde: debates atuais Ciência \& Saúde Coletiva, $13,2133$.

Mota, Roberta, \& David, Helena Maria. (2010). The increasing schooling of the community health agent: An induction of the work process? Trab Educ Saúde, 8(2), 19.

Nunes, Mônica de Oliveira , Trad, Leny Bonfim , Almeida, Bethânia de Araújo, Homem, Carolina Ramos, \& Melo, Marise Claudia I. de C. (2002). Community-based health workers: Building the identity of this hybrid, polyphonic character. Cadernos de Saúde Pública, 18(6), 8

The EconomicTimes. (2013). India, brazil help facebook expand user base to 1.11 bn. Retrieved 27/06/2013, 2013, from http://articles.economictimes.indiatimes.com/2013-05-06/news/39065047 1 registered-facebook-user-monthly-active-users-mobile-maus

Veletsianos, G. and Kimmons, R. (2013). Scholars and faculty members' lived experiences in online social networks. Internet and Higher Education, 16, 43-50.

Veletsianos, G., Kimmons, R., \& French, K. D. (2013). Instructor experiences with a social networking site in a higher education setting: Expectations, frustrations, appropriation, and compartmentalization. Etr\&D-Educational Technology Research and Development, 61(2), 255278. 